\title{
Chest CT imaging features and severity scores as biomarkers for prognostic prediction in patients with COVID-19
}

\author{
Shuchang Zhou ${ }^{1 \#}$, Chengyang Chen ${ }^{2 \#}$, Yiqi Hu${ }^{1}$, Wenzhi $\mathrm{Lv}^{3}$, Tao $\mathrm{Ai}^{1}$, Liming Xia ${ }^{1}$ \\ ${ }^{1}$ Department of Radiology, Tongji Hospital, Tongji Medical College, Huazhong University of Science and Technology, Wuhan, China; ${ }^{2}$ Department \\ of Radiology, The First Hospital of Hebei Medical University, Shijiazhuang, China; ${ }^{3}$ Department of Artificial Intelligence, Julei Technology \\ Company, Wuhan, China \\ Contributions: (I) Conception and design: T Ai; (II) Administrative support: L Xia; (III) Provision of study materials or patients: T Ai, L Xia; (IV) \\ Collection and assembly of data: C Chen, Y Hu; (V) Data analysis and interpretation: S Zhou, W Lv; (VI) Manuscript writing: All authors; (VII) \\ Final approval of manuscript: All authors. \\ \#These authors contributed equally to this work. \\ Correspondence to: Tao Ai, MD, PhD. Department of Radiology, Tongji Hospital, 1095 Jiefang Avenue, Qiaokou District, Wuhan 430030, China. \\ Email: aitao007@hotmail.com.
}

Background: Coronavirus disease 2019 (COVID-19) has become a pandemic. Few studies have explored the role of chest computed tomography (CT) features and severity scores for prognostic prediction. In this study, we aimed to investigate the role of chest CT severity score and imaging features in the prediction of the prognosis of COVID-19 patients.

Methods: A total of 134 patients (62 recovered and 72 deceased patients) with confirmed COVID-19 were enrolled. The clinical, laboratory, and chest CT (316 scans) data were retrospectively reviewed. Demographics, symptoms, comorbidities, and temporal changes of laboratory results, CT features, and severity scores were compared between recovered and deceased groups using the Mann-Whitney U test and logistic regression to identify the risk factors for poor prognosis.

Results: Median age was 48 and 58 years for recovered and deceased patients, respectively. More patients had at least one comorbidity in the deceased group than the recovered group (60\% vs. 29\%). Leukocytes, neutrophil, high-sensitivity C-reactive protein (hsCRP), prothrombin, D-dimer, serum ferritin, interleukin (IL)-2, and IL-6 were significantly elevated in the deceased group than the recovered group at different stages. The total CT score at the peak stage was significantly greater in the deceased group than the recovered group (20 vs. 11 points). The optimal cutoff value of the total CT scores was 16.5 points, achieving $69.4 \%$ sensitivity and $82.2 \%$ specificity for the prognostic prediction. The crazy-paving pattern and consolidation were more common in the deceased patients than those in the recovered patients. Linear opacities significantly increased with the disease course in the recovered patients. Sex, age, neutrophil, IL-2, IL-6, and total CT scores were independent risk factors for the prognosis with odds ratios of 3.8 to 8.7.

Conclusions: Sex (male), older age (>60 years), elevated neutrophil, IL-2, IL-6 level, and total CT scores $(\geq 16)$ were independent risk factors for poor prognosis in patients with COVID-19. Temporal changes of chest CT features and severity scores could be valuable for early identification of severe cases and eventually reducing the mortality rate of COVID-19.

Keywords: Coronavirus disease; computed tomography; severity score; risk factor; prognosis

Submitted Apr 19, 2020. Accepted for publication Oct 10, 2020.

doi: 10.21037/atm-20-3421

View this article at: http://dx.doi.org/10.21037/atm-20-3421 


\section{Introduction}

A novel pneumonia named coronavirus disease 2019 (COVID-19) was first reported in December 2019 and rapidly spread around the world. Subsequently, the World Health Organization (WHO) announced COVID-19 outbreak as a pandemic on March 12, 2020. As of August 19,2020 , a total of 21,938,207 confirmed cases, and 775,582 deaths were reported globally (1). The estimated mortality was $3.54 \%$, which was much lower than the earliest epicenter of Wuhan in China (about 6\%) and the subsequent epicenter of Italy in Europe (about 13\%). So far, the United States of America has the largest number of COVID-19 cases (cumulative number of 5,393,138 cases as of August 19, 2020), but the mortality rate (about 3.14\%) remains slightly below the average (2). Currently, the spread of COVID-19 is still getting worse in North America, South America, and South Asia, especially in the countries like United States of America, Brazil, and India.

Since there are no specific antiviral drugs and vaccines in the current stage, it is essential to identify severe and critically ill patients with COVID-19 at the early stage and give them timely supportive treatments to reduce the mortality. Several studies (3-7) reported that older age, comorbidities (cardiovascular and cerebrovascular diseases), and elevated serum levels of D-dimer, high-sensitivity C-reactive protein (hsCRP), and decreased lymphocytes were important high-risk factors associated with an increase of mortality rate in severe patients with COVID-19. What is more, the severity of the disease is also related to some special conditions, such as tumors and pregnancy $(8,9)$. Furthermore, the computed tomography (CT) severity score of lung involvement may also be related to the mortality of patients with COVID-19, which was reported from a preliminary study with a small sample size (10). However, few studies have been done to compare the clinical and chest CT features between the recovered and deceased patients with COVID-19 to quantitatively identify the risk factors for poor prognosis.

In this study, we aimed to comprehensively investigate the clinical, laboratory, and chest CT imaging features as risk factors for the prognostic prediction in patients with COVID-19. The information could be helpful for a better understanding of the disease pathophysiology, risk stratification, and early interventional plan-making, and eventually reduced the mortality. We present the following article in accordance with the STROBE reporting checklist (available at http://dx.doi.org/10.21037/atm-20-3421).

\section{Methods}

\section{Patients}

The study was conducted in accordance with the Declaration of Helsinki (as revised in 2013). This retrospective study was approved by our hospital institutional review boards (IRB; No. TJ-C20200141), and written informed consent was waived. From February 6 to 29, 2020, a total of 134 patients with COVID-19 confirmed by reverse transcription-polymerase chain reaction (RTPCR) tests with throat swab samples were enrolled in this study, including 62 recovered patients (recovered group) and 72 deceased patients (deceased group).

\section{Clinical information and laboratory results}

The clinical information and laboratory results of the patients were extracted from the electronic medical records in the hospital information system (HIS), including the baseline of demographics, symptoms after onset, and comorbidities. Laboratory test results included initial and follow-up blood routine tests, serum biochemical tests [alanine aminotransferase (ALT), aspartate aminotransferase (AST), albumin, urea, and creatine], inflammation-related factors [hsCRP, erythrocyte sedimentation rate (ESR), serum ferritin (SF), interleukin (IL)-2, IL-6 and tumor necrosis factor- $\alpha(\mathrm{TNF}-\alpha)$, and coagulation function [prothrombin time (PT) and D-dimer] during hospitalization.

\section{Chest CT imaging}

Non-contrast chest CT scans were performed for all patients using uCT 780 (United Imaging, Shanghai, China), GE lightspeed VCT (GE Healthcare, Milwaukee, WI, USA), or Siemens SOMATOM Definition AS+ (Siemens Healthcare, Forchheim, Germany). The patient was positioned in supine with breath-holding after deep inhalation during the scan. The scanning parameters were as follows: tube voltage $120 \mathrm{kV}$, automatic tube current modulation (40 mA), pitch $0.99-1.22 \mathrm{~mm}$, matrix $512 \times 512$, slice thickness $5-10 \mathrm{~mm}$, field of view, $350 \mathrm{~mm} \times 350 \mathrm{~mm}$, reconstruction slice thickness 0.625 or $1.25 \mathrm{~mm}$.

\section{Image interpretation}

A total of 316 CT scans from 134 patients (229 from the recovered and 87 from the deceased group) were evaluated by two experienced radiologists by consensus (Y.Q.H. and 
S.C.Z., with 8- and 13-year experience on thoracic imaging, respectively). Both readers were blinded to the clinical and laboratory information. All CT images were reviewed with the following window settings: lung window, widow width of 1,000-1,500 $\mathrm{HU}$ and window level of -700 to $550 \mathrm{HU}$; mediastinal window, window width of 300-350 HU, and window level of 30-40 HU. The main CT findings included ground-glass opacity (GGO), consolidation, crazy-paving pattern, vacuolar sign, and linear opacities.

The severity of lung involvement was visually scored according to the method described in previous studies (11). Briefly, a 5-point scale was used to quantitatively evaluate the extent of inflammatory pulmonary lesions for each of the five lung lobes: 0 , no lesions; $1,1-5 \%$ involvement; 2 , $\leq 25 \%$ involvement; $3,26-50 \%$ involvement; $4,51-75 \%$ involvement; 5, 76-100\% involvement. The total CT score of bilateral lungs was equal to the sum of the scores of five lobes, with a maximum total score of 25 points.

\section{Statistical analysis}

Statistical analysis was performed using the software SPSS for Windows (version 22.0; SPSS Inc., Chicago, IL, USA). Continuous variables were expressed as mean \pm standard deviation (SD) or median with interquartile range (IQR). Categorical variables were expressed as counts and percentages. The statistical differences in clinical features and chest $\mathrm{CT}$ features between survivors and non-survivors were compared using the Chi-squared test, or Fisher's exact test, as appropriate.

The disease course of each patient was divided into four stages: stage 1, week 1 after onset; stage 2, week 2 after onset; stage 3 , week 3 after onset; stage 4 , week 4 and more after onset (if possible). The laboratory test results from different stages were summarized for each parameter in survivors, as compared with the results immediately after the admission in non-survivors, using the Mann-Whitney U test.

The highest severity scores and severity scores summed up from different disease stages of lung involvement based on chest CT imaging were compared between survivors and non-survivors for the bilateral lungs (total CT score) and each of the five lung lobes, using the Mann-Whitney $\mathrm{U}$ test. A receiver-operating characteristic curve (ROC) analysis was applied to evaluate the accuracy of total CT severity scores for predicting the prognosis of patients with COVID-19. An optional cut-off value was further defined by Youden's index.

Multivariable logistic regression and lasso regression were performed to evaluate the relationship of clinical information, laboratory parameters, and CT score with the poor prognosis. The predictive ability of the logistic regression model was evaluated using ROC analysis.

A $\mathrm{P}<0.05$ was considered statistically significant. In addition, GraphPad Prism (version 6.0; GraphPad Software Inc., San Diego, CA, USA) was used for graphing.

\section{Results}

\section{Demographic and baseline clinical characteristics}

In the cohort of this study, the median age was 48 years (range, 14-84 years) and 58 years (range, 33-95 years) for survivors and non-survivors, respectively. The proportion of men was significantly higher in non-survivors than that in survivors $(\mathrm{P}<0.01)$. There were more patients with at least one underlying disease in non-survivors than that in survivors $(60 \%$ vs. $29 \% ; \mathrm{P}<0.001)$. The median time interval from illness onset to death was 15 days (range, 12-22 days) in non-survivors, which was significantly shorter than the disease duration in survivors (median, 32 days; range, 24-37 days). More detailed information is listed in Table 1.

\section{Comparisons of laboratory test results}

Since the time from illness onset to hospital admission was about 8 days, the admission time of the deceased patients was roughly equivalent to stage $2-3$ of the recovered patients. The levels of almost all the parameters of laboratory tests, except ALT and ESR, were significantly elevated in the deceased group, as compared with the results in the recovered group at different disease stages (all $\mathrm{P}<0.01$; Table 2 and Figure 1).

\section{Comparisons of chest CT imaging}

When selecting the chest CT images with peak severity from both groups, the total CT score of lung involvement was significantly greater in the deceased patients than that in the recovered patients $\{20[15-23] v s .11[7-15]$ in median value with IQR; $\mathrm{P}<0.001\}$, as well as the $\mathrm{CT}$ scores for each of the five lung lobes (all $\mathrm{P}<0.001$ ). The crazypaving pattern and vacuolar sign on chest CT images were significantly more commonly observed in the deceased patients than those in the recovered patients (all $\mathrm{P}<0.05$, except for the right lower lobe). By contrast, GGO and linear opacities were more commonly observed in the lower lobe of the bilateral lungs in the recovered patients (Table 3). 
Table 1 Demographic and clinical features of patients with COVID-19

\begin{tabular}{|c|c|c|c|}
\hline Features & $\begin{array}{l}\text { Recovered group } \\
(n=62)\end{array}$ & $\begin{array}{l}\text { Deceased group } \\
(n=72)\end{array}$ & $P$ values \\
\hline Age, years & 48 [38-61] & 68 [59-76] & $<0.0001$ \\
\hline Range & $14-84$ & 33-95 & \\
\hline Sex & & & 0.0049 \\
\hline $\mathrm{F}$ & $31(50 \%)$ & $18(25 \%)$ & \\
\hline M & $31(50 \%)$ & 54 (75\%) & \\
\hline $\begin{array}{l}\text { Fever (temperature } \\
\geq 37.3^{\circ} \mathrm{C} \text { ) }\end{array}$ & $52(84 \%)$ & 63 (88\%) & 0.7247 \\
\hline Cough & $16(26 \%)$ & $43(60 \%)$ & 0.0002 \\
\hline Sputum & $1(2 \%)$ & $11(15 \%)$ & 0.0139 \\
\hline Asthma & $6(10 \%)$ & $14(19 \%)$ & 0.1806 \\
\hline Dyspnea & $1(2 \%)$ & $17(24 \%)$ & 0.0005 \\
\hline Myalgia & $4(6 \%)$ & $2(3 \%)$ & 0.4147 \\
\hline Diarrhea & $0(0 \%)$ & $7(10 \%)$ & 0.0150 \\
\hline Comorbidity & $18(29 \%)$ & $43(60 \%)$ & 0.0007 \\
\hline Hypertension & $9(15 \%)$ & $29(40 \%)$ & 0.0019 \\
\hline Diabetes & $6(10 \%)$ & $12(17 \%)$ & 0.3529 \\
\hline $\begin{array}{l}\text { Coronary heart } \\
\text { disease }\end{array}$ & $4(6 \%)$ & $12(17 \%)$ & 0.1209 \\
\hline Carcinoma & $0(0 \%)$ & $5(7 \%)$ & 0.0613 \\
\hline $\begin{array}{l}\text { Chronic kidney } \\
\text { disease }\end{array}$ & $0(0 \%)$ & $8(11 \%)$ & 0.0074 \\
\hline Other & $3(5 \%)$ & $6(8 \%)$ & 0.5042 \\
\hline $\begin{array}{l}\text { Time from illness } \\
\text { onset to hospital } \\
\text { admission, days }\end{array}$ & 7 [4-10] & 8 [8-8] & 0.1153 \\
\hline $\begin{array}{l}\text { Time from illness } \\
\text { onset to discharge } \\
\text { (or death), days }\end{array}$ & 32 [24-37] & 15 [12-22] & $<0.0001$ \\
\hline
\end{tabular}

Data are median [IQR], $\mathrm{n}(\%)$. $\mathrm{P}$ values were calculated by MannWhitney $U$ test, $\chi^{2}$ test, or Fisher's exact test, as appropriate.

When comparing CT scores and imaging features at different disease stages of the two groups, the total CT severity scores were significantly greater in the deceased patients as compared with the recovered patients for the corresponding disease stage 1 to 3 (all $\mathrm{P}<0.001$ ). The statistical differences in CT scores of each of the five lobes were also observed between the two groups. For the analysis of imaging features, GGO and consolidation reached a peak

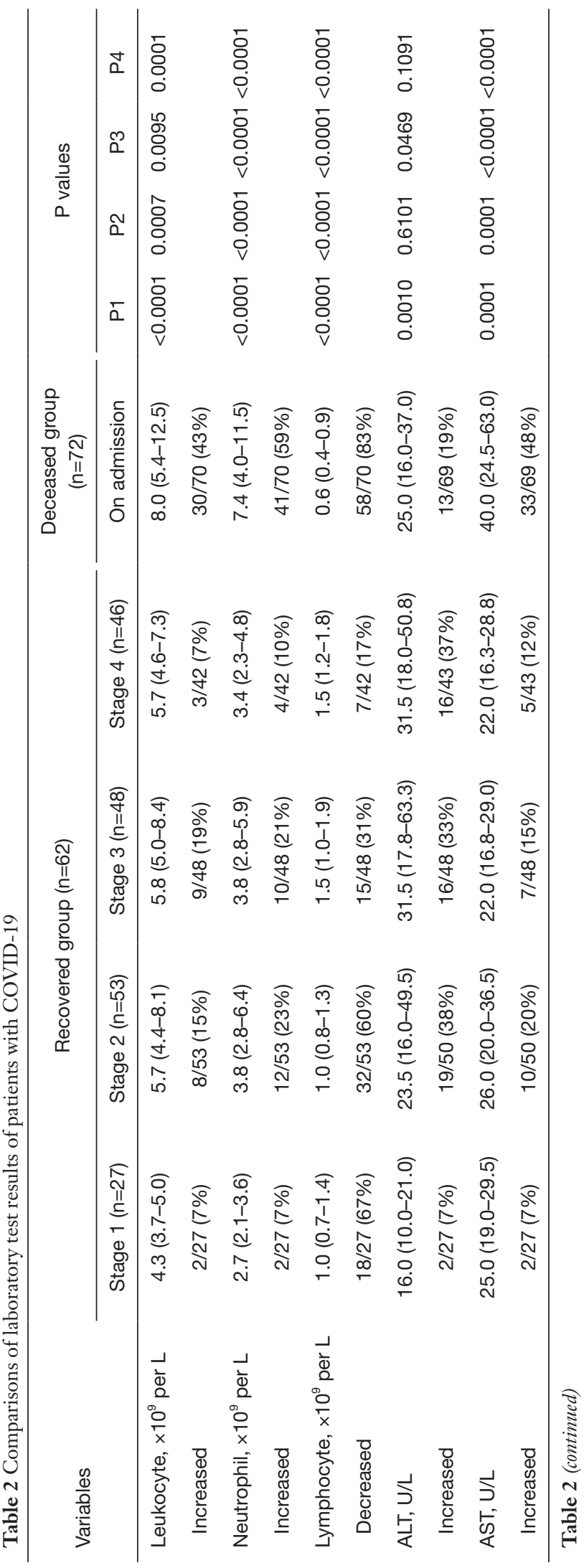




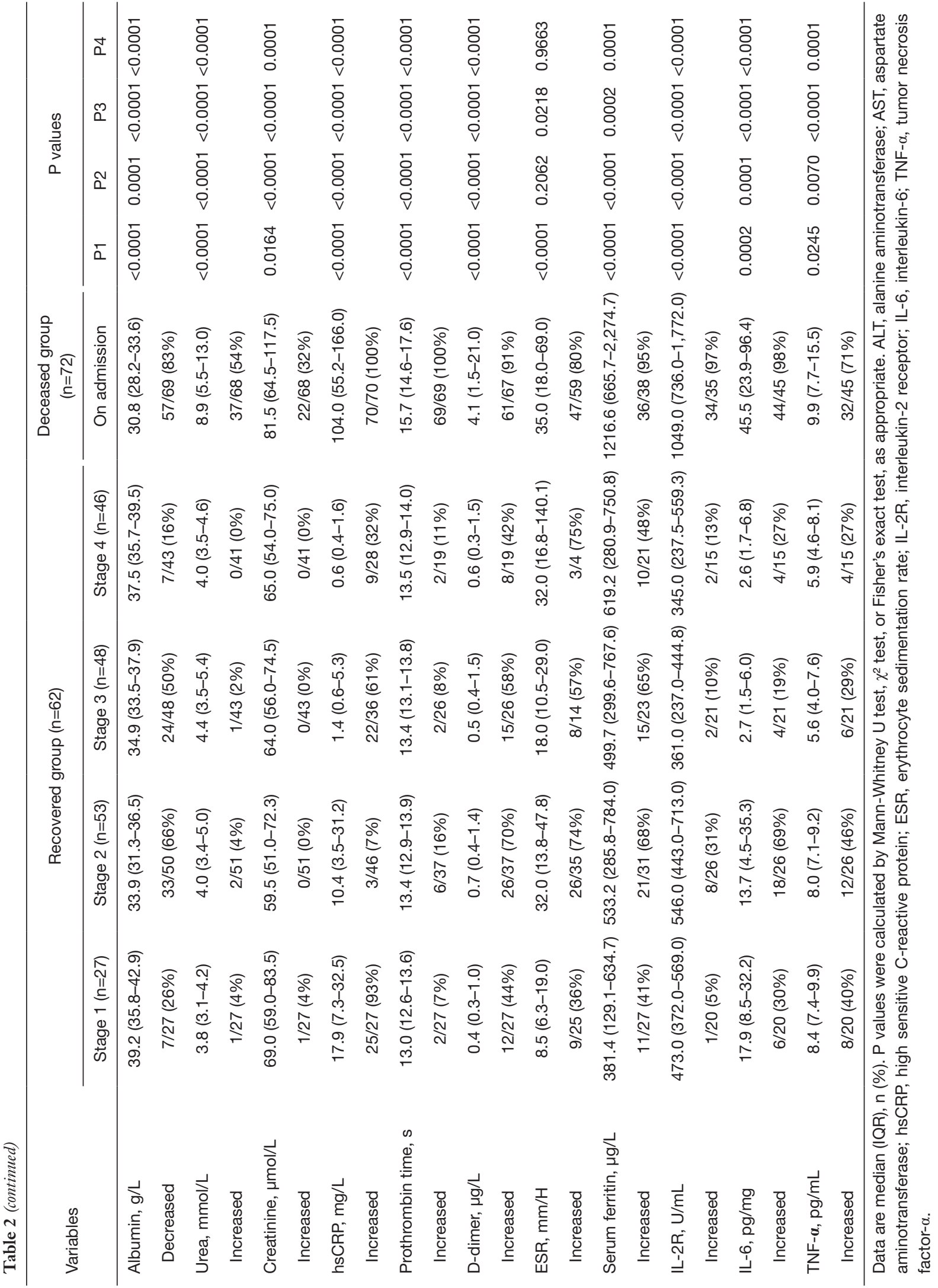



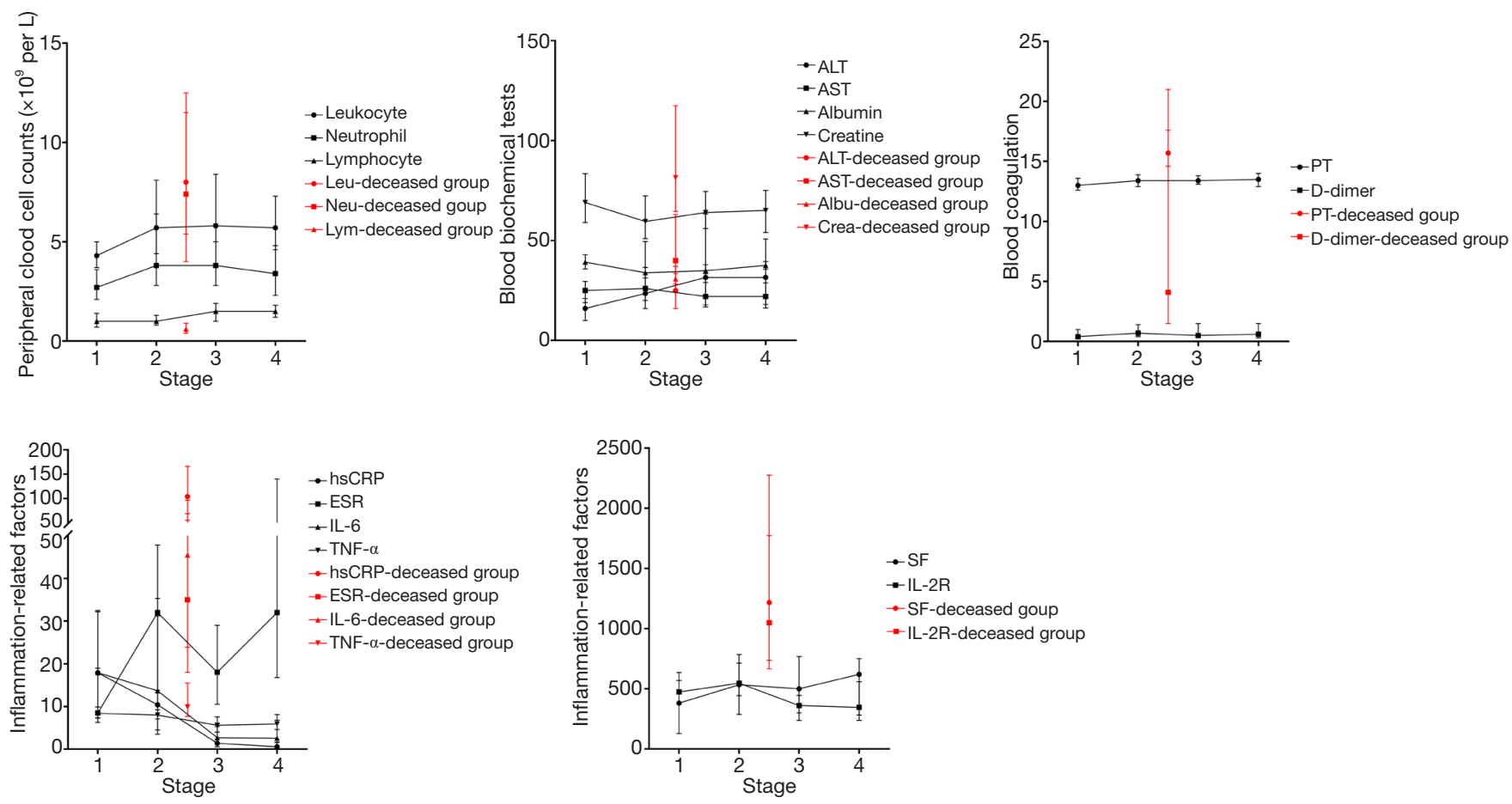

Figure 1 Temporal changes of laboratory test results (median with interquartile range) in the recovered group from stage 1 to 4 after the illness onset, as compared with the results of the deceased group immediately after the hospital admission (red symbols).

at stage 2 or 3 in the recovered patients; and linear opacities significantly increased with the disease course. However, in the group of deceased patients, consolidation was markedly increased with the disease course, without an obvious increase of linear opacities (Table 4 and Figures 2-5).

ROC analysis showed the area under the curve (AUC) of total CT severity scores was 0.817 (95\% CI, 0.744-0.890). The cut-off value of total CT scores was determined to be 16.5 points by Youden's index, which achieved $69.4 \%$ sensitivity and $82.3 \%$ specificity for predicting poor prognosis in patients with COVID-19.

\section{Multivariable logistic regression analysis}

The multiple variables of sex, age, hypertension, coronary heart disease, neutrophil, lymphocyte, ALT, IL-2, IL-6, TNF- $\alpha, \mathrm{D}$-dimer, and total CT score were finally selected for the modeling and calculation of odds ratios (ORs). The results showed that sex $(\mathrm{OR} 4.9, \mathrm{P}<0.01)$, age (OR 7.1, $\mathrm{P}=0.001)$, neutrophil count (OR 3.8, $\mathrm{P}<0.05)$, IL-2 (OR 8.2, $\mathrm{P}=0.001)$, IL-6 (OR 8.7, $\mathrm{P}<0.05)$ and total CT severity scores (OR 6.9, $\mathrm{P}=0.001)$ were independent risk factors for the prognosis of patients with COVID-19 (Table 5). The
Table 3 The comparisons of the highest CT scores of lung involvement and the corresponding imaging features between recovered and deceased patients

\begin{tabular}{lccc}
\hline Variables & $\begin{array}{c}\text { Recovered } \\
\text { group }(\mathrm{n}=62)\end{array}$ & $\begin{array}{c}\text { Deceased } \\
\text { group }(\mathrm{n}=72)\end{array}$ & P value \\
\hline Right upper lobe & & & \\
CT score & $2[1-3]$ & $4[3-5]$ & $<0.0001$ \\
GGO & $28(0 \%)$ & $25(35 \%)$ & 0.2914 \\
Consolidation & $22(35 \%)$ & $29(40 \%)$ & 0.6955 \\
Crazy-paving pattern & $26(42 \%)$ & $58(81 \%)$ & $<0.0001$ \\
Vacuolar sign & $9(15 \%)$ & $32(44 \%)$ & 0.0004 \\
Linear opacities & $12(19 \%)$ & $19(26 \%)$ & 0.4488 \\
Right middle lobe & & & \\
CT score & $1[0-3]$ & $4[2-5]$ & $<0.0001$ \\
GGO & $26(42 \%)$ & $24(33 \%)$ & 0.3967 \\
Consolidation & $18(29 \%)$ & $21(29 \%)$ & 1.0000 \\
Crazy-paving pattern & $16(26 \%)$ & $53(74 \%)$ & $<0.0001$ \\
Vacuolar sign & $3(5 \%)$ & $20(28 \%)$ & 0.0010 \\
Linear opacities & $15(24 \%)$ & $13(18 \%)$ & 0.5103 \\
\hline
\end{tabular}

Table 3 (continued) 
Table 3 (continued)

\begin{tabular}{|c|c|c|c|}
\hline Variables & $\begin{array}{c}\text { Recovered } \\
\text { group }(n=62)\end{array}$ & $\begin{array}{c}\text { Deceased } \\
\text { group }(n=72)\end{array}$ & $P$ value \\
\hline \multicolumn{4}{|l|}{ Right lower lobe } \\
\hline CT score & $3[2-4]$ & $5[4-5]$ & $<0.0001$ \\
\hline GGO & $29(47 \%)$ & $16(22 \%)$ & 0.0048 \\
\hline Consolidation & $39(63 \%)$ & $39(54 \%)$ & 0.3971 \\
\hline Crazy-paving pattern & $36(58 \%)$ & $53(74 \%)$ & 0.0861 \\
\hline Vacuolar sign & $13(21 \%)$ & $32(44 \%)$ & 0.0072 \\
\hline Linear opacities & $34(55 \%)$ & $20(28 \%)$ & 0.0026 \\
\hline \multicolumn{4}{|l|}{ Left upper lobe } \\
\hline CT score & $2[1-3]$ & 3 [3-4] & $<0.0001$ \\
\hline GGO & $36(58 \%)$ & $24(33 \%)$ & 0.0070 \\
\hline Consolidation & $18(29 \%)$ & $18(25 \%)$ & 0.7417 \\
\hline Crazy-paving pattern & $34(55 \%)$ & $53(74 \%)$ & 0.0367 \\
\hline Vacuolar sign & $6(10 \%)$ & $38(53 \%)$ & $<0.0001$ \\
\hline Linear opacities & $23(37 \%)$ & $13(18 \%)$ & 0.0224 \\
\hline \multicolumn{4}{|l|}{ Left lower lobe } \\
\hline CT score & $3(2-4]$ & $4(3-5]$ & $<0.0001$ \\
\hline GGO & $33(53 \%)$ & $16(22 \%)$ & 0.0004 \\
\hline Consolidation & $29(47 \%)$ & $28(39 \%)$ & 0.4561 \\
\hline Crazy-paving pattern & $33(53 \%)$ & $55(76 \%)$ & 0.0085 \\
\hline Vacuolar sign & $9(15 \%)$ & $32(44 \%)$ & 0.0004 \\
\hline Linear opacities & $38(61 \%)$ & $24(33 \%)$ & 0.0022 \\
\hline Total CT score & $11[7-15]$ & 20 [15-23] & $<0.0001$ \\
\hline
\end{tabular}

Data are median [IQR], $\mathrm{n}(\%)$. $\mathrm{P}$ values were calculated by MannWhitney $\mathrm{U}$ test, $\chi^{2}$ test, or Fisher's exact test, as appropriate.
AUC was 0.952 (95\% CI, 0.903-0.983, $\mathrm{P}<0.001)$ for the predictive ability of the prognostic model.

\section{Discussion}

In this study, we investigated a large amount of clinical, laboratory and CT features of 62 recovered and 72 deceased patients with COVID-19 to identify the risk factors for the poor prognosis. The results demonstrated that sex, age, neutrophil, IL-2, IL-6, and total CT severity scores were significantly correlated with the increased risk of poor prognosis in patients with COVID-19 (ORs 3.8-8.7, all $\mathrm{P}<0.05)$. In particular, the total CT scores were significantly higher in the deceased patients as compared with the recovered patients with COVID-19 at any disease stage \{median [IQR], 20 [15-23] vs. 11 [7-15] at peak stage, $\mathrm{P}<0.001\}$. The diagnostic performance of total CT scores achieved an AUC of 0.817 for the discrimination of the clinical outcomes (survival or non-survival). When comparing the patients with total CT scores of 16 points or more to the patients with less than 16 points, the risk of poor prognosis increased by 6.9 times. The cutoff value of 16.5 points based on CT severity achieved $69.4 \%$ sensitivity and $82.3 \%$ specificity for predicting poor prognosis in patients with COVID-19. In addition, chest CT features also had different patterns and trends in the recovered patients and deceased patients with COVID-19.

Although previous studies have reported several potential treatments for COVID-19 $(12,13)$, there are still no specific treatments and vaccines. Therefore, it was essential to identify severe and critically ill patients

Table 4 The comparisons of CT scores for different stages after the illness onset and the corresponding imaging features between recovered and deceased patients

\begin{tabular}{|c|c|c|c|c|c|c|c|c|c|c|}
\hline Variables & \multicolumn{4}{|c|}{ Recovered group (229 CT scans) } & \multicolumn{3}{|c|}{ Deceased group (87 CT scans) } & \multicolumn{3}{|c|}{$P$ value } \\
\hline CT score & $0[0-2]$ & 2 [0-3] & $1[0-2]$ & $2[1-3]$ & $3[2-4]$ & $4[3-4]$ & $5[4-5]$ & $<0.0001$ & $<0.0001$ & 0.0001 \\
\hline GGO & $16(32 \%)$ & $21(43 \%)$ & $27(51 \%)$ & $36(47 \%)$ & $20(42 \%)$ & 7 (24\%) & $3(30 \%)$ & 0.4338 & 0.1552 & 0.3078 \\
\hline Crazy-paving pattern & $12(24 \%)$ & $20(41 \%)$ & $13(25 \%)$ & $26(34 \%)$ & $32(67 \%)$ & $26(90 \%)$ & 7 (70\%) & 0.0001 & 0.0001 & 0.0084 \\
\hline Vacuolar sign & $4(8 \%)$ & $7(14 \%)$ & $2(4 \%)$ & $2(3 \%)$ & $17(35 \%)$ & $17(59 \%)$ & $2(20 \%)$ & 0.0022 & 0.0001 & 0.1151 \\
\hline Linear opacities & $2(4 \%)$ & $10(20 \%)$ & $18(34 \%)$ & $39(51 \%)$ & $10(21 \%)$ & $7(24 \%)$ & $3(30 \%)$ & 0.0255 & 0.9189 & 1.0000 \\
\hline
\end{tabular}

Table 4 (continued) 
Table 4 (continued)

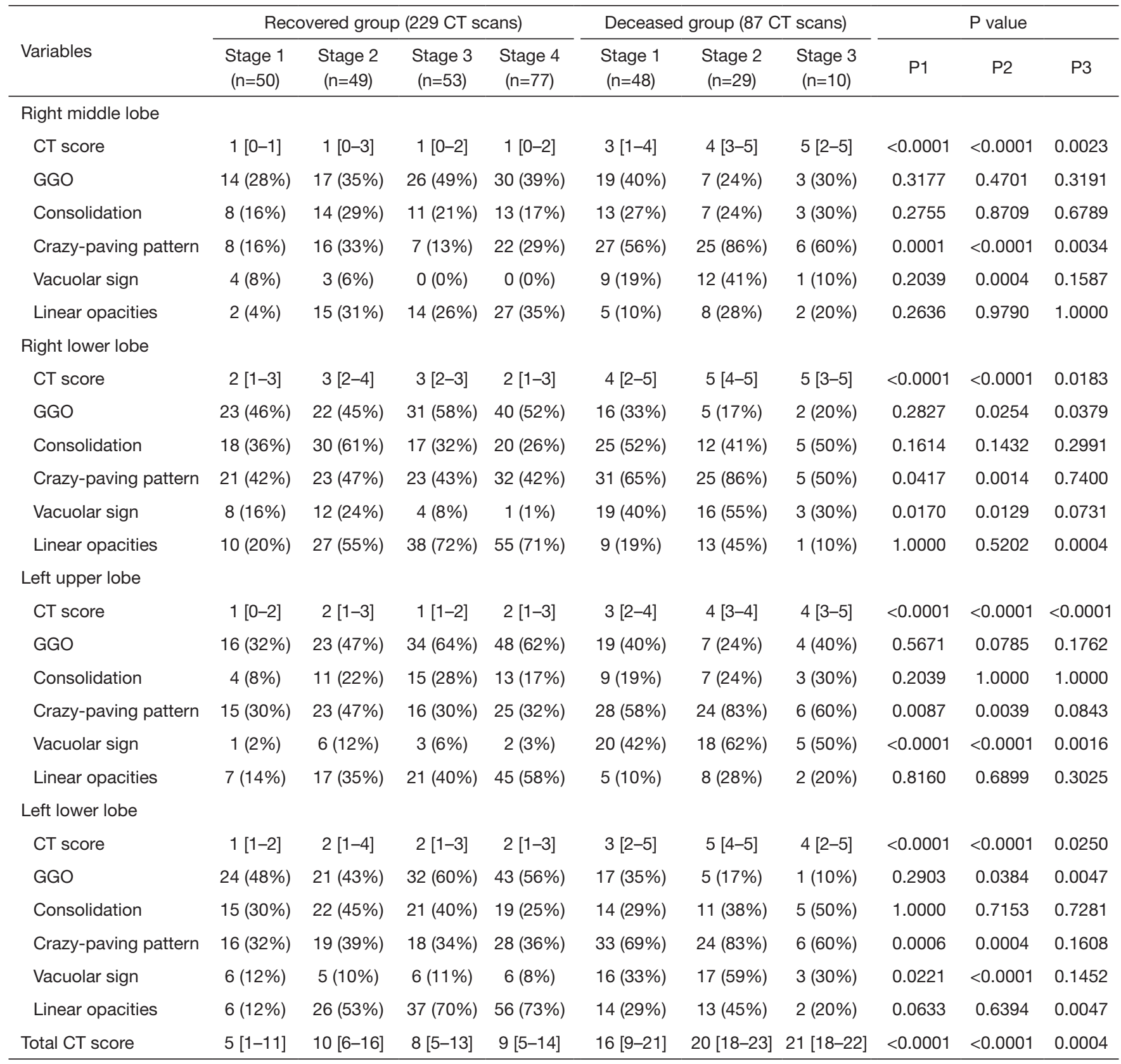

Stages 1-4 mean the periods of $1^{\text {st }}$ week, $2^{\text {nd }}$ week, $3^{\text {rd }}$ week and more than 3 weeks after the illness onset. Data are median [IQR], $n(\%)$. $P$ values were calculated by Mann-Whitney U test, $\chi^{2}$ test, or Fisher's exact test, as appropriate. GGO, ground-glass opacity.

with COVID-19 at an early stage and provide timely supportive therapy to reduce the mortality rate. Yang et al. reported that older patients ( $>65$ years) with comorbidities were at increased risk of death (14). Wu et al. reported that older age, lymphopenia, multiple organ dysfunction, and coagulation dysfunction were closely associated with disease deterioration and death (15). In the cohort of this study, the median age was significantly older in the deceased patients than that in the recovered patients \{median [range], 68 [59-76] vs. 48 [38-61], $\mathrm{P}<0.001$ \}; the proportion of patients with underlying diseases had increased significantly in the deceased group as compared 


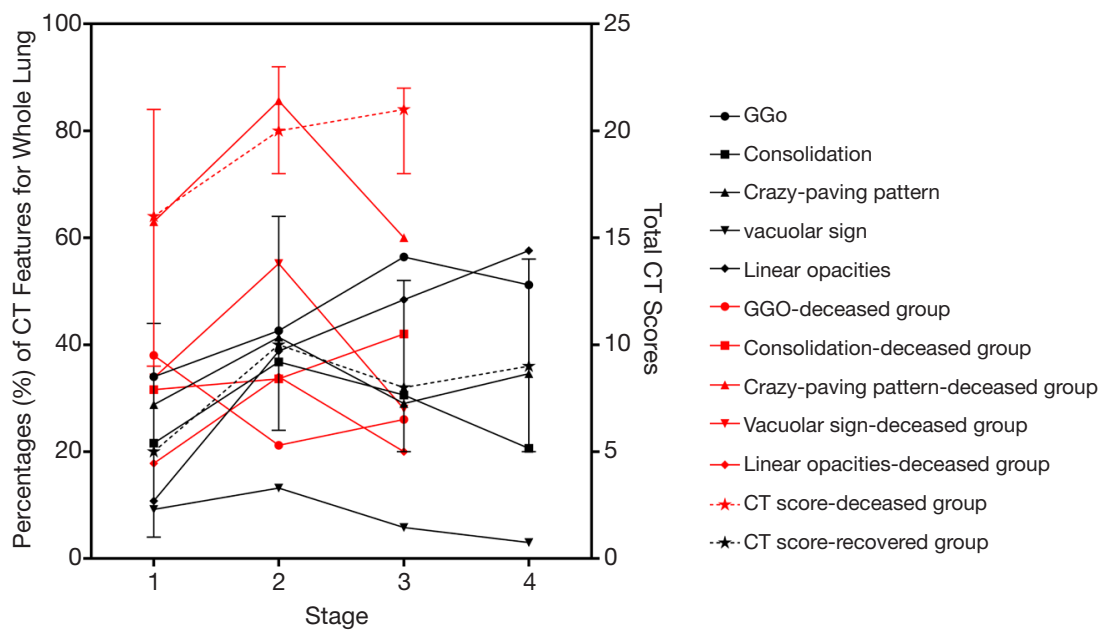

Figure 2 Temporal changes of chest CT features (percentages averaged by the five lobes) and severity scores (median with interquartile range) between the recovered group and deceased group (red symbols) at the different disease stages.

with the recovered group $(60 \%$ vs. $29 \%, \mathrm{P}<0.001)$, which was consistent with previous studies.

The pathogenesis of highly pathogenic and highly infectious human coronavirus remains unclear. The virusinduced cytokine storm is thought to play important roles in disease severity. It has been proven that both severe acute respiratory syndrome (SARS) and Middle Eastern respiratory syndrome (MERS) patients had high levels of serum pro-inflammatory cytokines, which increased the accumulation of macrophages and neutrophils in the lung and peripheral blood, resulting in extensive lung injury (16). In this study, the levels of neutrophils, serum IL-2 and IL-6 were significantly elevated in the deceased patients as compared with the recovered patients, which were also identified as independent risk factors of the prognosis. Considering the significantly increased level of hsCRP in serum, subsequent bacterial pulmonary infection after the virus invasion may also play an important role in aggravating lung injury and suddenly deteriorating of COVID-19.

In addition, liver and kidney dysfunction and coagulation disorder were reported to be observed in COVID-19 patients by several recent studies $(3,15,17)$. In this study, however, most patients had normal liver, kidney, and coagulation functions in the recovered group. In the deceased group, serum ALT and creatinine were elevated only in $19 \%$ and $32 \%$ of the patients, respectively. A previous pathological study reported only moderate microvascular steatosis and mild lobular and portal activity in a COVID-19 patient with acute respiratory distress syndrome (ARDS) (18), and angiotensin-converting enzyme 2 (ACE-2), a receptor of SARS-CoV-2, was mainly expressed but extremely low in bile duct epithelial cells in the normal liver tissues (19) and the proximal tubules of the kidney (20). These results indicated that hypoxic ischemia or drug-induced liver/kidney injury may be the main mechanisms of liver and kidney dysfunction, instead of a direct attack by the virus.

Although previous studies have reported some correlations between imaging features and disease severity, there was limited evidence to support the hypothesis that denser or more confluent radiological lung involvement is linked to worse clinical outcomes (21). Zhang et al. demonstrated that the chest CT score had a positive association with inflammation indicators and was a good indicator of the extent of systemic inflammation (22). Qin et al. found that some specific CT features (peripheral distribution of lesions and crazy-paving pattern) could improve the efficiency of differential diagnosis between COVID-19 pneumonia and non-COVID-19 pneumonia (23). So far, no study has revealed the prognostic value of CT scores on the severity of this disease. In this study, we preliminarily identified the CT imaging features and severity scores as independent risk factors of the poor prognosis in patients with COVID-19. Several studies have described the imaging features and temporal changes of chest CT in patients with COVID-19 $(11,24,25)$. The main CT features in this study included GGO, consolidation, crazy-paving pattern, vacuolar sign, and linear opacities, as described in the previous studies $(11,26,27)$. However, the results showed that crazy- 


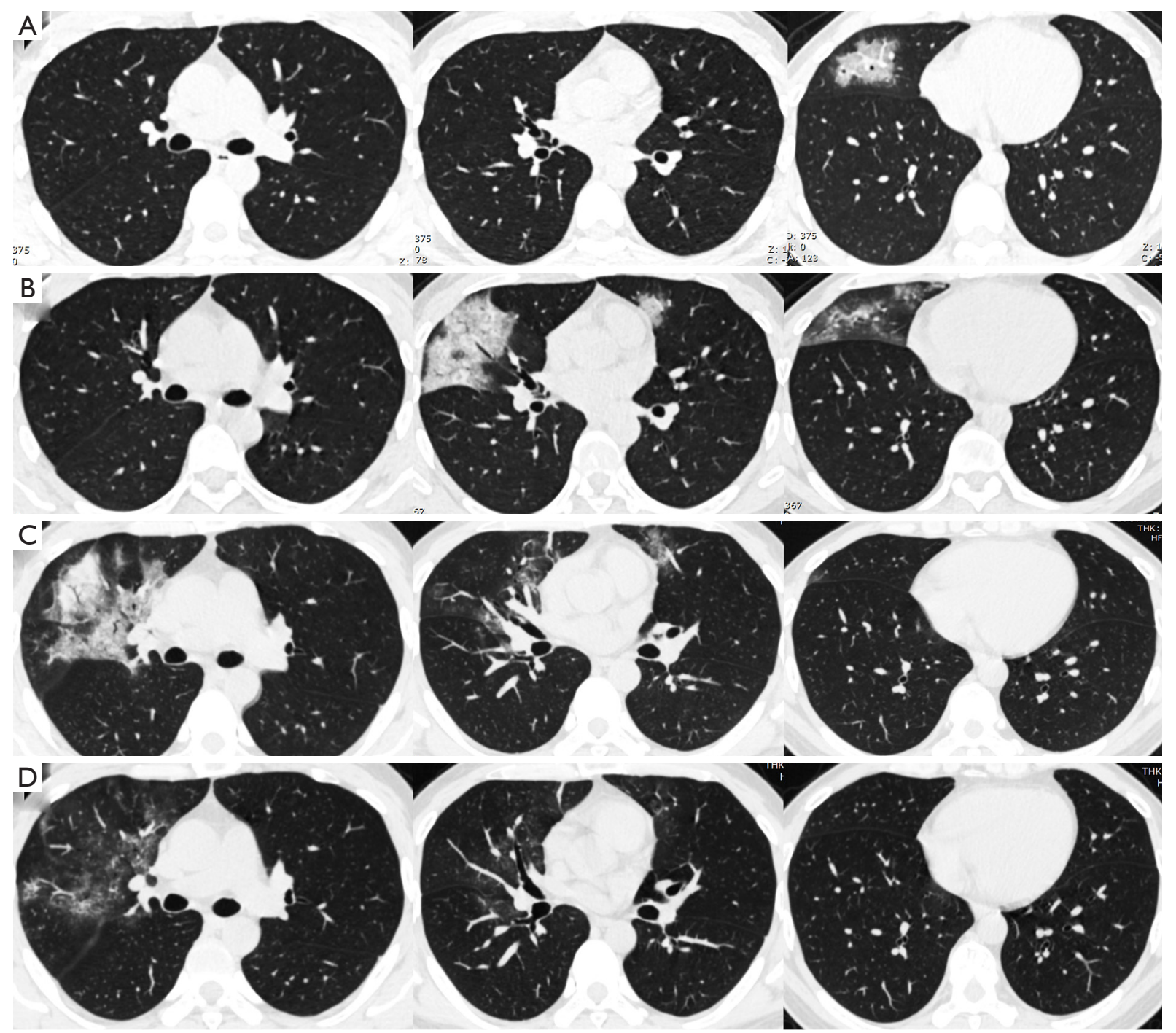

Figure 3 A 34-year-old man with fever and dry cough for about half a month. (A) Initial chest CT obtained 4 days after the onset showed only focal consolidation in the middle lobe of the right lung (total CT score, 2); (B) 7 days later, the first follow-up chest CT showed the consolidation was getting worse, and an additional lesion was observed adjacent to the heart in the left upper lung (total CT score, 6). (C) 7 days later, the second follow-up chest CT showed the increased extent of the diseases in the right lung; however, the consolidation was partially absorbed in bilateral lungs (total CT score, 11). (D) 10 days later (28 days after the illness onset), the third follow-up CT showed marked absorption of the lung diseases in bilateral lungs, and only minor mixed GGO opacities were observed in the right middle lobe (total CT score, 6). GGO, ground-glass opacity.

paving pattern and vacuolar sign were significantly more commonly observed in the deceased patients than those in the recovered patients, while GGO and linear opacities were more commonly observed in the lower lobes of bilateral lungs in the recovered patients. Crazy-paving pattern and vacuolar sign reflect severe infiltration of lung interstitium and consequent inflammation of small blood vessels, which may be highly suggestive of a disease progression (25). GGO occurred at the early stage of the disease may be caused by the partial filling of airspaces due to inflammatory exudation; however, at the late (absorption) stage, it could be caused by a combination of interstitial thickening, partial collapse of alveoli, and incomplete absorption of inflammatory exudation. The results showed 

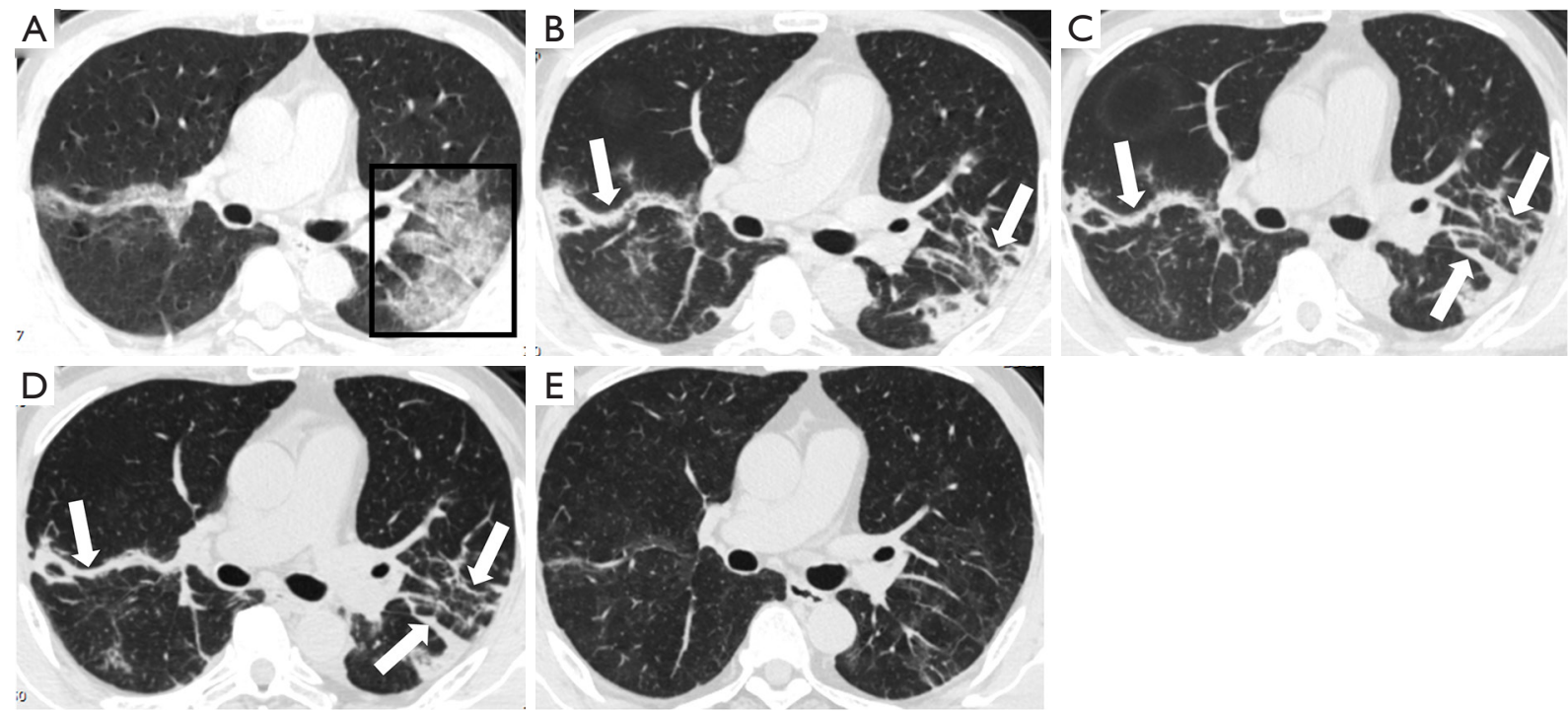

Figure 4 A 61-year-old man with cough and asthenia for 14 days. (A) Band-like GGO in the lower lobe of the right lung, and patchy GGO with interlobular septal thickening (box) in the left lung on day 14 after the onset. (B,C,D) The lesions were gradually absorbed with the increase of linear opacities (white arrows) from days 14 to 31. (E) Only a few linear opacities remained on day 58 after the illness onset. GGO, ground-glass opacity.
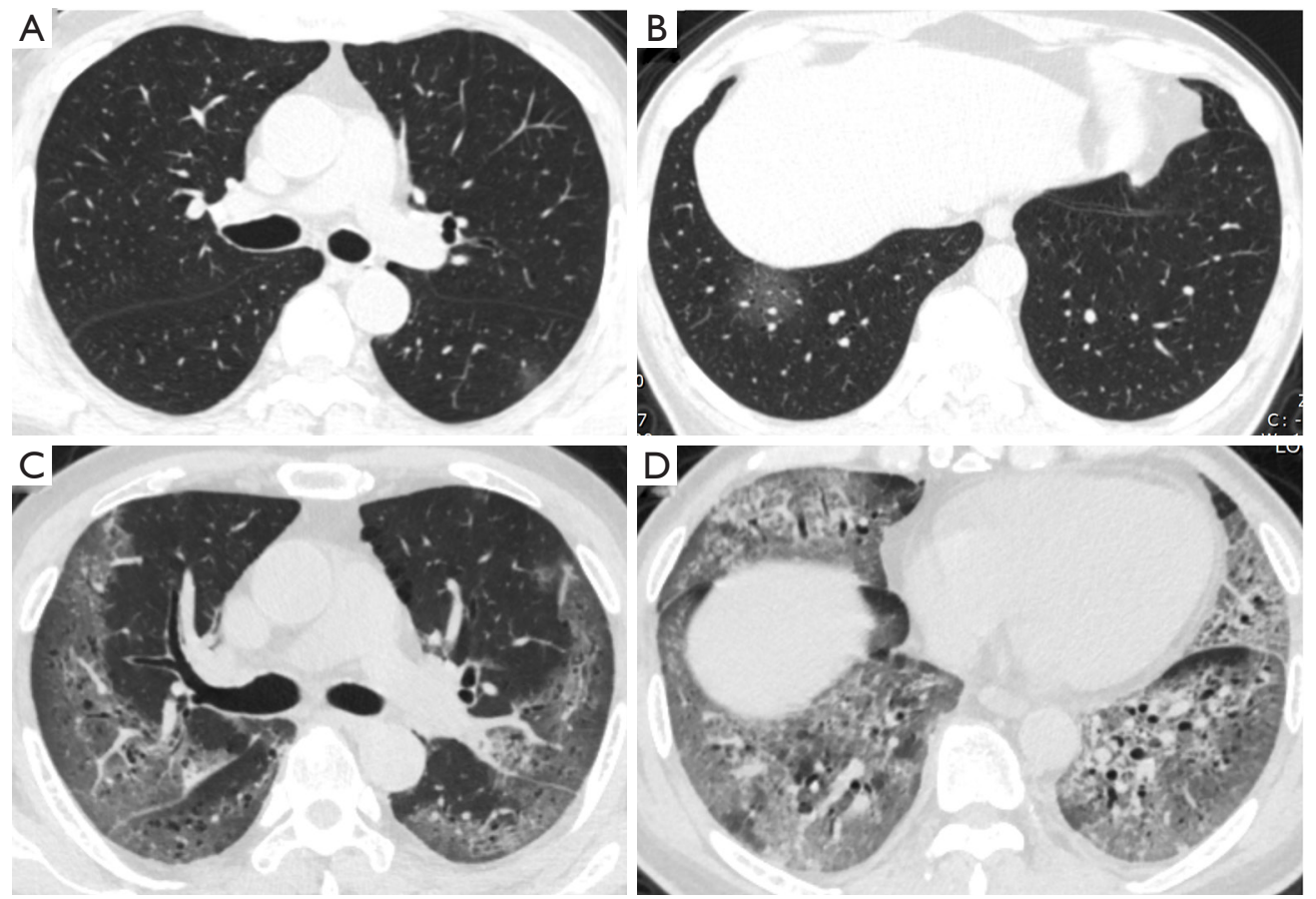

Figure 5 A 59-year-old man with fever, dyspnea, dry cough for 10 days. (A,B) small patchy GGO in the lower lobes of bilateral lungs on day 1 after the onset (total CT score, 3). (C,D) The disease rapidly progressed, appearing as diffuse GGO in bilateral lungs, with interlobular septal thickening and focal consolidation on day 9 (total CT score, 21). The patient died 9 days after the last CT scan. GGO, ground-glass opacity. 
Table 5 The results of multivariable logistic regression analysis

\begin{tabular}{|c|c|c|c|}
\hline Factors & Levels & Odds ratios $(95 \% \mathrm{Cl})$ & $P$ value \\
\hline ALT, U/L & $>41$ vs. $\leq 41$ & $0.144(0.034-0.611)$ & 0.009 \\
\hline Sex & Male vs. female & $4.904(1.489-16.157)$ & 0.009 \\
\hline $\begin{array}{l}\text { Neutrophil count, } \\
\times 10^{9} \text { per } L\end{array}$ & $>6.3$ vs. $\leq 6.3$ & $3.834(1.133-12.970)$ & 0.031 \\
\hline Total CT score & $\geq 16$ vs. $<16$ & 6.877 (2.133-22.179) & 0.001 \\
\hline $\mathrm{IL}-2 \mathrm{R}, \mathrm{U} / \mathrm{mL}$ & $>710$ vs. $\leq 710$ & $8.223(2.577-26.238)$ & 0.001 \\
\hline IL-6, pg/mg & $\geq 7.0$ vs. $<7.0$ & $8.723(1.440-52.820)$ & 0.018 \\
\hline Age, years & $\geq 60$ vs. $<60$ & 7.059 (2.201-22.633) & 0.001 \\
\hline $\mathrm{TNF}-\alpha, \mathrm{pg} / \mathrm{mL}$ & $\geq 8.1$ vs. $<8.1$ & $0.644(0.181-2.299)$ & 0.498 \\
\hline $\begin{array}{l}\text { Lymphocyte } \\
\text { count, } \times 10^{9} \text { per } L\end{array}$ & $\geq 1.1$ vs. $<1.1$ & $0.757(0.180-3.180)$ & 0.704 \\
\hline Hypertension & Yes vs. no & $0.820(0.192-3.496)$ & 0.789 \\
\hline $\begin{array}{l}\text { Coronary heart } \\
\text { disease }\end{array}$ & Yes vs. no & $1.098(0.202-5.959)$ & 0.914 \\
\hline D-dimer, $\mu \mathrm{g} / \mathrm{L}$ & $>0.5$ vs. $\leq 0.5$ & $2.339(0.477-11.464)$ & 0.295 \\
\hline
\end{tabular}

ALT, alanine aminotransferase; IL-2R, interleukin-2 receptor; IL-6, interleukin-6; TNF- $\alpha$, tumor necrosis factor- $\alpha$.

no obvious statistical differences between the recovered and deceased groups based on the analysis of different stages. Therefore, GGO may not be a good imaging characteristic for the evaluation of the disease severity and prognosis. Interestingly, linear opacity was significantly increased with the disease course in the recovered group (from $10.80 \% \pm 6.14 \%$ at stage 1 to $57.60 \% \pm 13.94 \%$ at stage 4 , averaged by five lobes); meanwhile, the proportion of linear opacity was from $17.80 \% \pm 7.19 \%$ at stage 1 to $20.00 \% \pm 6.32 \%$ at stage 3 in the deceased group. The results indicated that the increase of linear opacities was associated with a better prognosis. In addition, consolidation was undoubtedly markedly increased with the disease course in the deceased group, reflecting a progressing involvement from lung interstitium to the parenchyma.

Several studies have demonstrated the application value of visual CT scores in evaluating disease severity, temporal changes, and prognosis $(10,28)$. The results of this study also showed that the CT severity scores were significantly greater in the deceased group than those in the recovered group for each of the five lobes and the whole lung. As an independent risk factor of the prognosis identified by multivariable logistic regression analysis, the mortality risk was increased by 6.9 times in patients with total CT scores $\geq 16$ points as compared with the patients with a total CT score $<16$ points. What is more, the optimal cutoff value of a total CT score of 16.5 points achieved $69.4 \%$ sensitivity and $82.2 \%$ specificity with an AUC of 0.817 (95\% CI, 0.744-0.890). These results strongly suggested that wellperformed visual CT severity scoring could be valuable in predicting the prognosis of early-stage COVID-19 patients.

There were some limitations in the current study. First, the sample size is relatively small, and this retrospective study was conducted in a single center; thus, a multicenter study with large sample size is needed for further validation. Second, most patients in the recovered group were mild to moderate illness; the predictive value of selected risk factors may be overestimated. Therefore, well-matched groups in disease severity should be investigated to more accurately evaluate the risk factors related to the mortality of COVID-19.

\section{Conclusions}

Sex (male), age ( $>60$ years), elevated levels of neutrophils, IL-2 and IL-6, and total CT severity scores $(\geq 16)$ were independent risk factors for poor prognosis in patients with COVID-19. Temporal changes of chest CT features and severity scores were closely associated with the mortality of COVID-19, which may be valuable for early identification of severe cases and eventually reducing the mortality rate of COVID-19.

\section{Acknowledgments}

Funding: None.

\section{Footnote}

Reporting Checklist: The authors have completed the STROBE reporting checklist. Available at http://dx.doi. org/10.21037/atm-20-3421

Data Sharing Statement: Available at http://dx.doi. org/10.21037/atm-20-3421

Peer Review File: Available at http://dx.doi.org/10.21037/ atm-20-3421

Conflicts of Interest: All authors have completed the ICMJE uniform disclosure form (available at http://dx.doi. org/10.21037/atm-20-3421). The authors have no conflicts 
of interest to declare.

Ethical Statement: The authors are accountable for all aspects of the work in ensuring that questions related to the accuracy or integrity of any part of the work are appropriately investigated and resolved. The study was conducted in accordance with the Declaration of Helsinki (as revised in 2013). This retrospective study was approved by our hospital institutional review boards (IRB; No. TJC20200141) and written informed consent was waived.

Open Access Statement: This is an Open Access article distributed in accordance with the Creative Commons Attribution-NonCommercial-NoDerivs 4.0 International License (CC BY-NC-ND 4.0), which permits the noncommercial replication and distribution of the article with the strict proviso that no changes or edits are made and the original work is properly cited (including links to both the formal publication through the relevant DOI and the license). See: https://creativecommons.org/licenses/by-nc-nd/4.0/.

\section{References}

1. World Health Organization. Available online: https:// covid19.who.int/?gclid=EAIaIQobChMI5M2gzM_76gI VRaqWCh140QAoEAAYASAAEgJhovD_BwE (accessed Aug 19, 2020).

2. Sahu KK, Kumar R. Current perspective on pandemic of COVID-19 in the United States. J Family Med Prim Care 2020;9:1784-91.

3. Zhou F, Yu T, Du R, et al. Clinical course and risk factors for mortality of adult inpatients with COVID-19 in Wuhan, China: a retrospective cohort study. Lancet 2020;395:1054-62.

4. Leung C. Clinical features of deaths in the novel coronavirus epidemic in China. Rev Med Virol 2020;30:e2103.

5. Mizumoto K, Chowell G. Estimating Risk for Death from Coronavirus Disease, China, January-February 2020. Emerg Infect Dis 2020;26:1251-6.

6. Ruan Q, Yang K, Wang W, et al. Clinical predictors of mortality due to COVID-19 based on an analysis of data of 150 patients from Wuhan, China. Intensive Care Med 2020;46:846-8.

7. Liu W, Tao ZW, Lei W, et al. Analysis of factors associated with disease outcomes in hospitalized patients with 2019 novel coronavirus disease. Chin Med J (Engl) 2020;133:1032-8.
8. Jindal V, Sahu KK, Gaikazian S, Siddiqui AD, Jaiyesimi I. Cancer treatment during COVID-19 pandemic. Med Oncol 2020;37:58.

9. Chen Y, Bai J. Maternal and infant outcomes of fullterm pregnancy combined with COVID-2019 in Wuhan, China: retrospective case series. Arch Gynecol Obstet 2020;302:545-51.

10. Yuan M, Yin W, Tao Z, et al. Association of radiologic findings with mortality of patients infected with 2019 novel coronavirus in Wuhan, China. PLoS One 2020;15:e230548.

11. Pan F, Ye T, Sun P, et al. Time Course of Lung Changes at Chest CT during Recovery from Coronavirus Disease 2019 (COVID-19). Radiology 2020;295:715-21.

12. Wang M, Cao R, Zhang L, et al. Remdesivir and chloroquine effectively inhibit the recently emerged novel coronavirus (2019-nCoV) in vitro. Cell Res 2020;30:269-71.

13. Roback JD, Guarner J. Convalescent Plasma to Treat COVID-19: Possibilities and Challenges. JAMA 2020;323:1561-2.

14. Yang $\mathrm{X}, \mathrm{Yu} \mathrm{Y}, \mathrm{Xu} \mathrm{J}$, et al. Clinical course and outcomes of critically ill patients with SARS-CoV-2 pneumonia in Wuhan, China: a single-centered, retrospective, observational study. Lancet Respir Med 2020;8:475-81.

15. Wu C, Chen X, Cai Y, et al. Risk Factors Associated With Acute Respiratory Distress Syndrome and Death in Patients With Coronavirus Disease 2019 Pneumonia in Wuhan, China. JAMA Intern Med 2020;180:934-43.

16. Kindler E, Thiel V. SARS-CoV and IFN: Too Little, Too Late. Cell Host Microbe 2016;19:139-41.

17. Chen T, Wu D, Chen H, et al. Clinical characteristics of 113 deceased patients with coronavirus disease 2019: retrospective study. BMJ 2020;368:m1091.

18. Xu Z, Shi L, Wang Y, et al. Pathological findings of COVID-19 associated with acute respiratory distress syndrome. Lancet Respir Med 2020;8:420-2.

19. Guan GW, Gao L, Wang JW, et al. Exploring the mechanism of liver enzyme abnormalities in patients with novel coronavirus-infected pneumonia. Zhonghua Gan Zang Bing Za Zhi 2020;28:E2.

20. Simoes ESA, Teixeira MM. ACE inhibition, ACE2 and angiotensin-(1-7) axis in kidney and cardiac inflammation and fibrosis. Pharmacol Res 2016;107:154-62.

21. Lal A, Mishra AK, Sahu KK. CT chest findings in coronavirus disease-19 (COVID-19). J Formos Med Assoc 2020;119:1000-1.

22. Zhang J, Meng G, Li W, et al. Relationship of chest 


\section{Page 14 of 14}

CT score with clinical characteristics of 108 patients hospitalized with COVID-19 in Wuhan, China. Respir Res 2020;21:180.

23. Qin L, Yang Y, Cao Q, et al. A predictive model and scoring system combining clinical and CT characteristics for the diagnosis of COVID-19. Eur Radiol 2020;30:6797807.

24. Shi H, Han X, Jiang N, et al. Radiological findings from 81 patients with COVID-19 pneumonia in Wuhan, China: a descriptive study. Lancet Infect Dis 2020;20:425-34.

25. Zhou S, Wang Y, Zhu T, et al. CT Features of Coronavirus

\section{Zhou et al. Chest CT for prognostic prediction with COVID-19}

Disease 2019 (COVID-19) Pneumonia in 62 Patients in Wuhan, China. AJR Am J Roentgenol 2020;214:1287-94.

26. Bernheim A, Mei X, Huang M, et al. Chest CT Findings in Coronavirus Disease-19 (COVID-19): Relationship to Duration of Infection. Radiology 2020;295:200463.

27. Li K, Wu J, Wu F, et al. The Clinical and Chest CT Features Associated with Severe and Critical COVID-19 Pneumonia. Invest Radiol 2020;55:327-31.

28. Li K, Fang Y, Li W, et al. CT image visual quantitative evaluation and clinical classification of coronavirus disease (COVID-19). Eur Radiol 2020;30:4407-16.

Cite this article as: Zhou S, Chen C, Hu Y, Lv W, Ai T, Xia L. Chest CT imaging features and severity scores as biomarkers for prognostic prediction in patients with COVID-19. Ann Transl Med 2020;8(21):1449. doi: 10.21037/atm-20-3421 